\title{
Sawtooth pacing with on-axis ICRH modulation in JET-ILW
}

\author{
E. Lerche ${ }^{1,2}$, M. Lennholm ${ }^{1}$, I. S. Carvalho ${ }^{1,3}$, P. Dumortier ${ }^{1,2}$, F. Durodie ${ }^{2}$, D. Van Eester ${ }^{2}$, \\ J. Graves $^{4}, \mathrm{Ph}$. Jacquet ${ }^{1}$, A. Murari ${ }^{5}$ and JET contributors ${ }^{[1]}$ \\ EUROfusion Consortium, JET, Culham Science Centre, Abingdon, OX14 3DB, UK \\ ${ }^{I}$ CCFE, Culham Science Centre, Abingdon, United Kingdom \\ ${ }^{2}$ LPP-ERM/KMS, Association EUROFUSION-Belgian State, TEC partner, Brussels, Belgium \\ ${ }^{3}$ Instituto de Plasmas e Fusão Nuclear, EUROFUSION-IST, University of Lisbon, Portugal \\ ${ }^{4}$ CRPP-EPFL, Association EUROFUSION - Confédération Suisse, Lausanne, Switzerland \\ ${ }^{5}$ Consorzio RFX, Association EUROFUSION - CNR/ENEA, Padova, Italy
}

\begin{abstract}
A novel technique for sawteeth control in tokamak plasmas using ion-cyclotron resonance heating (ICRH) has been developed in the JET-ILW tokamak [2]. Unlike previous ICRH methods, which explored the destabilization of the internal kink mode when the radiofrequency (RF) wave absorption was placed near the $q=1$ surface, the technique presented here consists of stabilizing the sawteeth as fast as possible by applying the ICRH power centrally and inducing a sawtooth crash by switching it off at the appropriate instant. Since it employs on-axis ICRH, this technique is aligned with other ICRH applications that require central plasma heating such as high-Z impurity control and fusion power enhancement. The validation of this method in JET-ILW L-mode discharges is presented.
\end{abstract}

\section{Introduction}

In tokamak plasmas with a central safety factor lower than unity $\left(q_{0}<1\right)$, the central plasma parameters commonly exhibit a non-linear oscillation, known as the sawtooth instability [3]. The sawtooth cycle manifests itself through a slow increase in the plasma temperature (and other parameters) in the central part of the plasma, followed by an abrupt crash, usually resetting the plasma profiles approximately to those at the start of the cycle. Large sawtooth crashes can, however, trigger more deleterious instabilities, such as neo-classical tearing modes (NTMs) [4,5]. Such modes will reduce the plasma confinement significantly and, if the instability grows sufficiently large, they can lead to a plasma disruption. Large sawtooth crashes following long quiescent periods without a crash have been seen to be much more likely to trigger NTMs than crashes associated with a shorter inter-crash interval. For this reason it is important to assure that the sawtooth period $\left(\tau_{S W T}\right)$ remains shorter than the threshold for NTM triggering. Nevertheless, excessive shortening of the sawtooth period does result in a moderate confinement reduction and for this reason it is desirable to maintain it as long as possible without inducing NTMs. It is therefore useful to develop methods to control the sawtooth period to a preset value for high performance scenario development.

A number of different methods have been developed to control sawteeth. The traditional actuators for implementing such control are Electron Cyclotron Resonance Heating and Current Drive (ECRH) and Ion Cyclotron Resonance Heating and Current Drive (ICRH).
ECRH can affect the sawtooth period by driving current near the $q=1$ surface, modifying the local current profile in this region [5-12]. In continuous control schemes, the ECRH power is kept constant while the ECRH deposition location is controlled in real-time to adjust the sawtooth period [8]. Alternatively the ECRH power can be modulated with a modulation period equal to the desired sawtooth period. Such control scheme is referred to in the literature as sawtooth pacing or sawtooth locking [10-12].

In addition to affecting the local current profile near $q=1$, ICRH influences the sawtooth period by modifying the pressure and the distribution function of the RF-heated ions in the plasma [13-14]. Recent work [15] has also highlighted the influence of the fast ion orbit topology near the $q=1$ surface on the sawtooth dynamics. Sawtooth control based on the modification of the fast ion distribution function near $q=1$ with ICRH has been demonstrated in JET [16-21], including real time control of the deposition location through variation of the RF frequency.

The current paper describes the first demonstration of sawtooth control through modulation of the ICRH power. The concept is similar to the ECRH pacing and locking experiments $[10,11]$, the difference being that the main effect of the ICRH modulation is to change the fast ion population properties (pressure, anisotropy, etc.) in the plasma centre rather than perturb the $q$-profile near the $q=1$ surface. Sawtooth pacing acting on the fast ion stabilization properties (local heating) is inherently slower than pacing based on $q$ - 
profile perturbations (local current-drive) since the slowing-down dynamics of the fast ions is part of the pacing process. The main advantage of the former technique is that it relies on central ICRH deposition rather than on off-axis power deposition $(q \sim 1)$ and therefore the location of the ICRH power is not critical as long as a sufficiently large fraction is deposited inside the $q=1$ surface. As a consequence the ICRH can be deposited at the optimal location required by other considerations, such as for avoiding central accumulation of heavy impurities in metallic machines [22-31] or for enhancing fusion power [32].

\section{Experimental results}

The first experiments for sawtooth control with on-axis ICRH modulation in JET-ILW were done in L-mode plasmas with $B_{0}=2.7 \mathrm{~T}, I_{\mathrm{P}}=2 \mathrm{MA}, n_{\mathrm{e} 0}=(5 \pm 0.5) \times 10^{19} / \mathrm{m}^{3}$. Central hydrogen minority ICRH at $f=42.5 \mathrm{MHz}$ (with dipole phasing) was used with $\mathrm{P}_{\text {icrh }}=3-4 \mathrm{MW}$. Figure 1 shows the comparison between a discharge with constant ICRF power (left) and two pulses with ICRH power modulation with $f_{\text {mod }}=3 \mathrm{~Hz}$ and $5 \mathrm{~Hz}$ (duty-cycle $75 \%$ ).
The non-modulated discharge is characterized by a sequence of long sawtooth build-ups followed by rapid crashes (b). The long sawteeth (with periods exceeding $0.5 \mathrm{~s}$ ) always trigger considerable magnet-hydro-dynamic (MHD) activity, though in this pulse not causing lasting NTMs (c). It is interesting to note that, while this mode is present, the sawteeth are shorter and the plasma performance is degraded (d). When the ICRH power is modulated in otherwise identical plasma conditions, the sawteeth are efficiently paced at the RF modulation frequency and the $N=2$ MHD activity is fully suppressed. Despite the $25 \%$ lower averaged ICRF power applied, the peak temperatures are comparable to the constant ICRH power case. The total plasma stored energy is also similar in the two discharges, probably due to the absence of $N=2$ MHD activity in the modulated case which partially compensates for the lower time averaged ICRH power applied. The insert in Fig.1b-right shows the details of the sawtooth pacing dynamics: The sawtooth crash is delayed with respect to the RF notch because it takes some time for the change in the fast particle population to affect the core electron dynamics as described in section 3 .
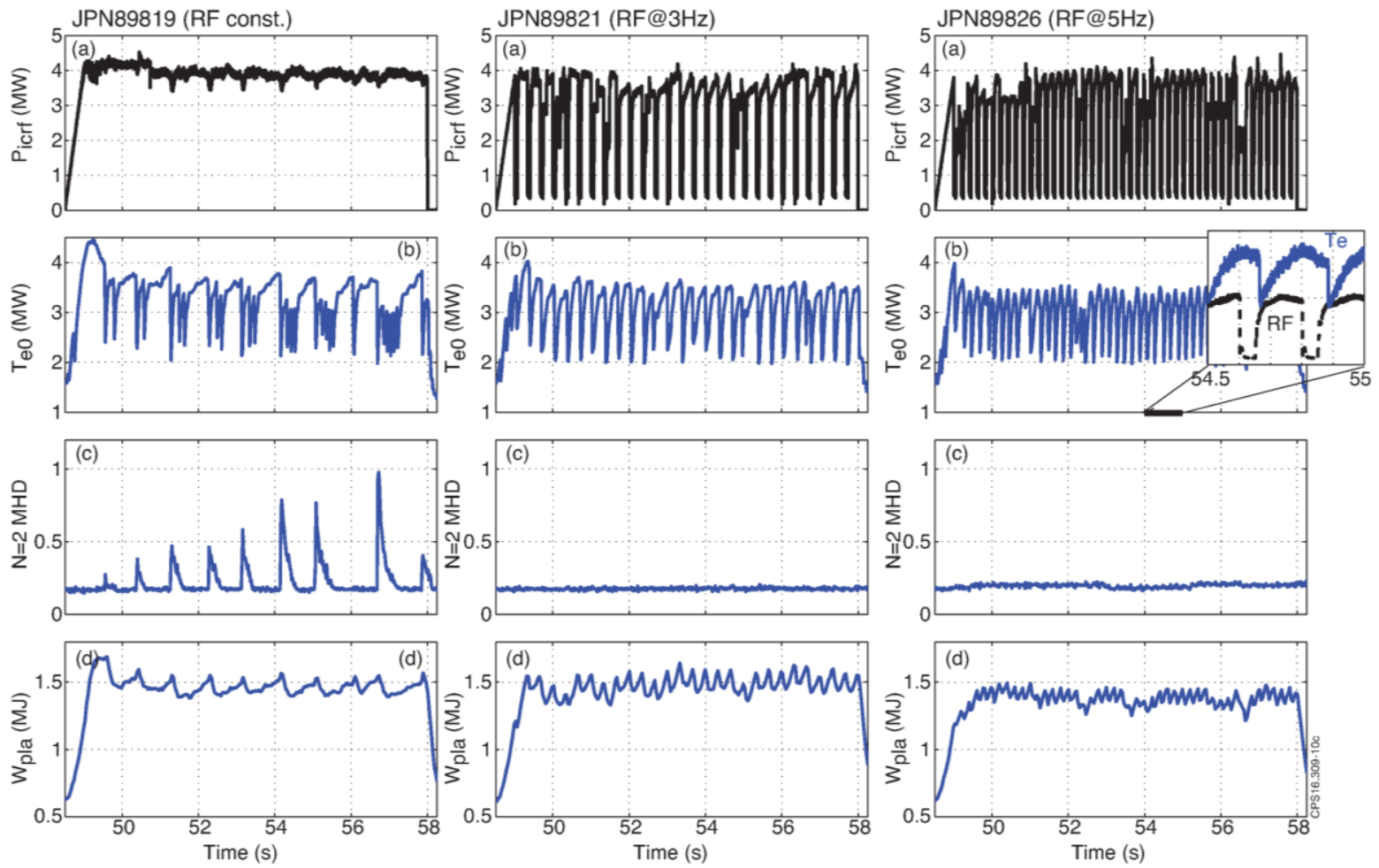

Fig.1: Comparison of three L-mode discharges with constant (89819), 3Hz (89281) and 5Hz (89826) modulated ICRF heating, illustrating the successful sawtooth pacing and total suppression of the N=2 MHD activity in the latter: (a) ICRF power; (b) central electron temperature; (c) N=2 MHD mode amplitude; (d) plasma stored energy.

One of the main objectives of these experiments was to determine the range of modulation frequencies that can be used to reliably pace sawteeth in JET plasmas. The main results are summarized in Fig.2, which shows the experimental sawtooth periods as function of time obtained in similar discharges with different RF modulation frequencies, $f_{\text {mod }}=0$ (RF const.), $2 \mathrm{~Hz}, 3 \mathrm{~Hz}$ and $5 \mathrm{~Hz}$, all with duty-cycle $=75 \%$. The maximum $\mathrm{RF}$ power was roughly constant $\left(P_{\text {icrf }} \sim 4 \mathrm{MW}\right)$ and the $\mathrm{H}$ concentration was similar $\left(n_{H} / n_{e}=2-3 \%\right)$ in all discharges.
In the non-modulated case one recognizes the composite sawteeth dynamics with long (0.5-0.7s) sawteeth interleaved with very short ones. When the ICRF power is modulated at $2 \mathrm{~Hz}$, the maximum sawtooth period is, as expected, reduced to $\tau_{S W T}=0.5 \mathrm{~s}$ but the shorter sawteeth still exist as the $N=2$ modes are not fully suppressed. In the $3 \mathrm{~Hz}$ and $5 \mathrm{~Hz} \mathrm{RF}$ modulation cases, the $N=2$ activity is absent and the large majority of the sawteeth follow the RF modulation frequency, illustrating the efficient sawteeth control obtained in 
these conditions. The relative number of RF-triggered sawteeth (normalized to the total number of sawteeth detected) increases with modulation frequency from $45 \%$ at $2 \mathrm{~Hz}$ to $70 \%$ and $85 \%$ in the $3 \mathrm{~Hz}$ and $5 \mathrm{~Hz}$ cases, respectively, since less spontaneous sawteeth occur in the latter. The triggering efficiency, defined as the number of RF-triggered sawteeth divided by the number of RF notches is high in all cases, $80 \%$ at $2 \mathrm{~Hz}, 83 \%$ at $3 \mathrm{~Hz}$ and $88 \%$ at $5 \mathrm{~Hz}$, and does not depend strongly on the RF modulation frequency in the studied conditions.
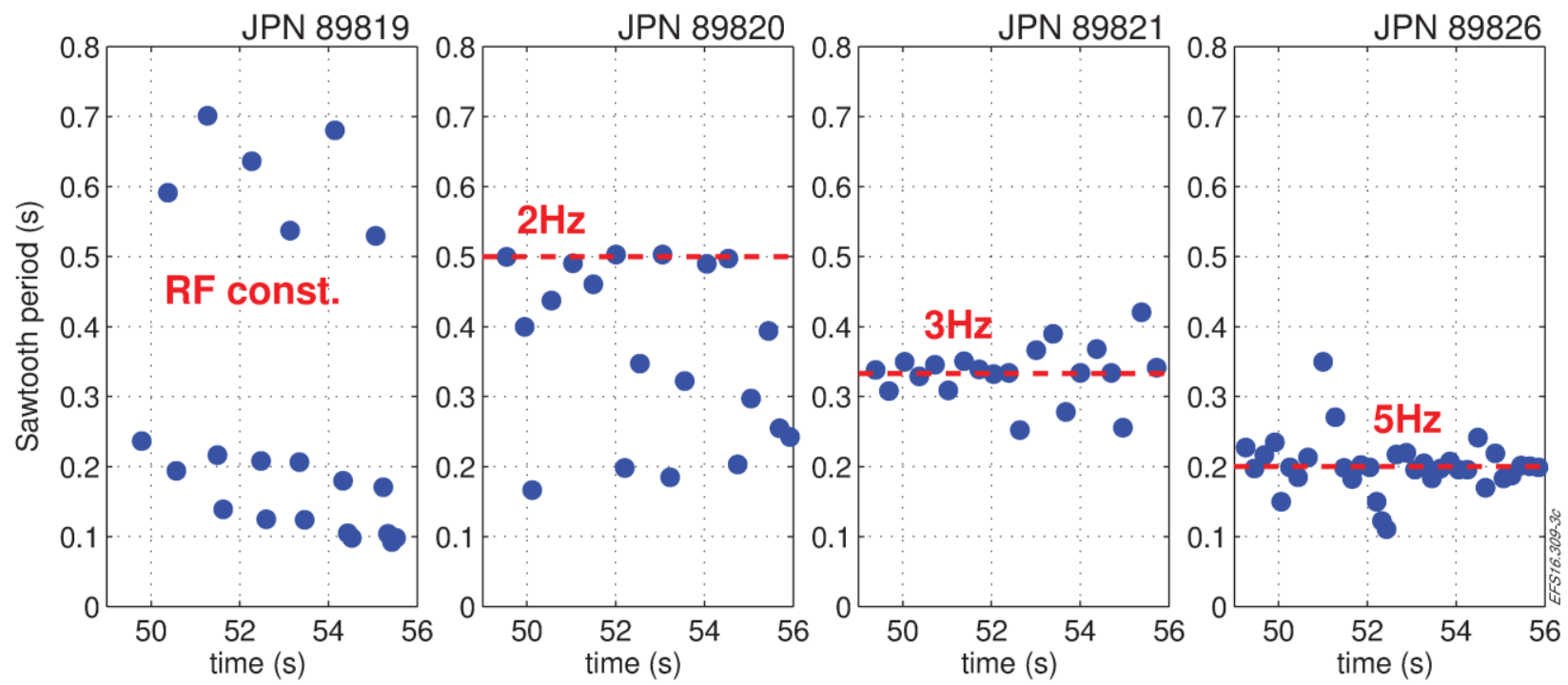

Fig. 2: Sawtooth period vs. time for 4 similar L-mode discharges with constant and modulated ICRF power.

\section{Time delays (slowing-down)}

Although the actual physics of sawtooth stabilisation by fast particles is quite complex and depends on details of the fast particle distribution function in specific regions of the plasma $[16,17]$, the basic characteristics of the described method can be illustrated via first principle plasma parameters such as the slowing down time of the RF-induced fast particles. The slowing-down time of the fast ions onto the electrons does not depend on the fast particle energy and is given by $\tau_{S D}=C .\left(k_{B} T_{e}\right)^{3 / 2} / n_{e}$ [33], where $n_{\mathrm{e}}$ and $T_{\mathrm{e}}$ are the local electron density and temperature, respectively, $k_{B}$ is the Boltzman's constant and $C$ is a constant that depends on the fast ion mass (protons in this case) and the Coulomb logarithm. Figure 3 shows the time intervals between the RF switch-off instants and the respective sawtooth crashes in an L-mode discharge with an RF modulation frequency of $3 \mathrm{~Hz}$. This pulse was run at $B_{0}=2.7 \mathrm{~T} /$ $I_{\mathrm{P}}=2 \mathrm{MA}$ with approximately $4 \mathrm{MW}$ of on-axis modulated $\mathrm{H}$ minority ICRH at $f=42.5 \mathrm{MHz}$ (dipole phasing). The peak core electron temperature was $T_{\mathrm{e} 0}=3.5 \mathrm{keV}$ and the central plasma density was $n_{\mathrm{e} 0}=5 \times 10^{19} / \mathrm{m}^{3}$. The dotted traces represent the electron slowing-down time $\tau_{S D}$ computed from the experimental $n_{\mathrm{e} 0}$ and $T_{\mathrm{e} 0}$ signals. The fluctuation in this trace is due to the variation of the central electron temperature during the sawtooth cycles. As expected from first order theory, the time delays between the RF switch-off instants and the sawtooth crashes are comparable to the slowing down time of the fast ions throughout the discharge.

The correlation between the slowing-down dynamics of the RF accelerated ions and the sawteeth triggering times is further illustrated in Fig.4, where the results of a statistical analysis of a series of L-mode discharges are shown. The curves represent the experimental distribution function of the waiting time from the start of the ICRH notch to the next sawtooth crash. This distribution time represents the relative number of triggering events detected in a narrow time window as function of the time delay between the ICRH power notches and the subsequent sawtooth crashes. The data are taken from a series of similar discharges (with different RF modulation frequencies). The statistical results confirm that the highest sawtooth triggering probability is indeed centred around the lower boundary of the average electron slowing-down time of the fast ions in these pulses, $\tau_{S D}=40-70 \mathrm{~ms}$. More sophisticated statistical analysis methods have also been employed and confirmed these observations [34].

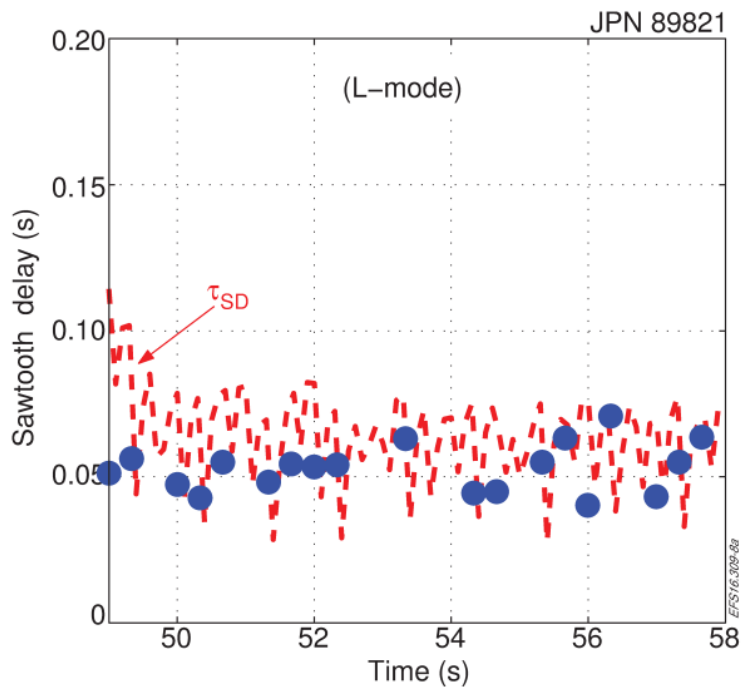

Fig. 3: Time delays between the RF switch-off instants and the sawtooth crashes for an L-mode discharge as function of time. $\mathrm{P}_{\text {icrh }}=4 \mathrm{MW}$ of central ICRH modulated at $3 \mathrm{~Hz}$ was applied. The dashed curve represents the fast ions slowing-down time. 


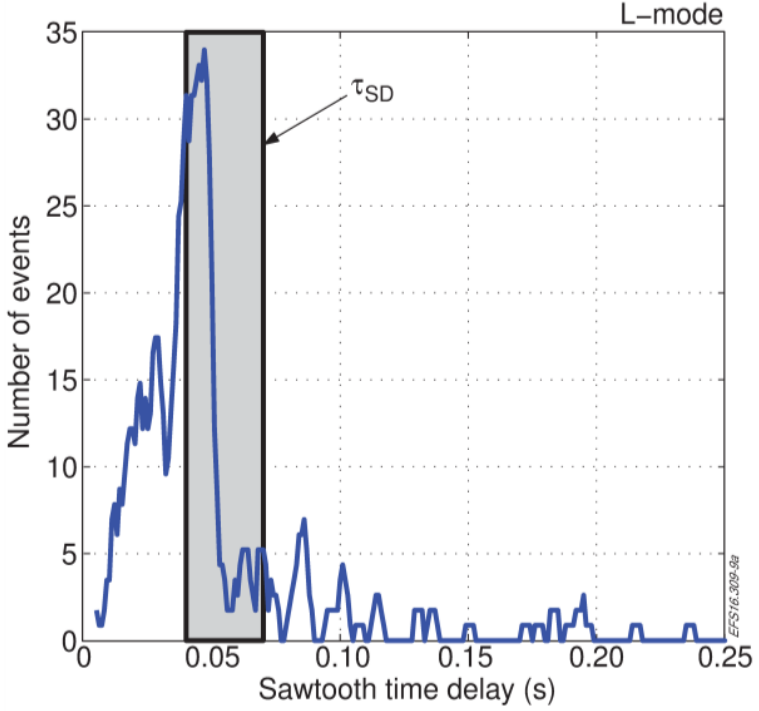

Fig. 4: Statistical analysis of the time delays between the RF switch-off instants and the sawtooth crashes for a series of Lmode discharges. The grey area represents the range of the slowing-down times of the fast ions in these pulses.

\section{Summary \& Discussion}

Reliable sawtooth control was achieved for the first time with modulated on-axis ICRF heating in JET-ILW Lmode discharges with $B_{0}=2.7 \mathrm{~T}, I_{\mathrm{P}}=2 \mathrm{MA}$. Central $\mathrm{H}$ minority ICRH at $f=42.5 \mathrm{MHz}$ (dipole phasing) with $P_{\text {icrh }}=3-4 \mathrm{MW}$ was used with modulation frequencies ranging from $2 \mathrm{~Hz}$ to $5 \mathrm{~Hz}$. While the non-modulated reference plasmas were characterized by long sawteeth $\left(\tau_{\mathrm{SWT}}>0.5 \mathrm{~s}\right)$ and considerable $N=2$ MHD activity following the sawtooth crashes, the discharges in which the sawteeth were paced with $f_{\text {mod }} \geq 3 \mathrm{~Hz}$ showed complete suppression of the $N=2$ modes. The triggering efficiency (number of RF-triggered sawteeth over number of RF notches) is typically above $80 \%$ in the cases studied. The lag time between the RF switch-off instant and the sawtooth crash is well correlated to the electron slowingdown time of the fast ions and does not depend on the RF modulation frequency in the studied range. A realtime control algorithm that imposes the RF power notch after a pre-programmed time interval has elapsed from the last detected sawtooth crash was also tested [35]. It showed remarkable pacing efficiency (close to $100 \%$ ) and it minimizes the number of notches and their duration thus maximizing the averaged ICRF power applied.

Preliminary studies (not described in this paper) indicate that this technique can also be applied to moderate NBI power H-modes $\left(B_{0}=2.7 \mathrm{~T}, I_{\mathrm{P}}=2 \mathrm{MA}\right.$, $\left.P_{\mathrm{NBI}} \sim 10 \mathrm{MW}\right)$ at modest ICRH power levels $(2-4 \mathrm{MW})$, despite the stabilizing contribution of the NBI ions [2]. In this case, reliable sawtooth pacing requires more careful tuning of the ICRF heating parameters (RF power, $\mathrm{H}$ conc.) according to the discharge properties, but once the optimal conditions are reached, the triggering efficiency is comparable to L-mode ( $\geq 80 \%)$. The delays between the RF switch-off instants and the sawtooth crashes also correlate with the slowing-down time of the fast ions, but there are indications that other effects - such as the NBI stabilization - contribute to the effective time delay for a sawtooth to be triggered. One important question is whether this technique can also be used in DT fusion plasmas, where the $\alpha$-particles (in addition to the NBI ions) also contribute to the background sawteeth stabilization. Numerical tools that properly describe the competition between the different fast ion sources taking into account their different particle distributions and the synergy between them are still under development. Another question is whether pacing the sawteeth at an optimal frequency is beneficial for minimizing high- $Z$ core impurity accumulation and how it compares with the impurity screening obtained by applying constant RF power on-axis (and not controlling the sawteeth).

\section{Acknowledgements}

This work has been carried out within the framework of the EUROfusion Consortium and has received funding from the EURATOM research and training programme 2014-2018 under grant agreement No 633053. The views and opinions expressed herein do not necessarily reflect those of the European Commission.

\section{References}

[1] "Overview of the JET results in support to ITER" by X. Litaudon et al., Proc of $16^{\text {th }}$ IAEA FEC (Kyoto, 2016)

[2] E. Lerche et al., Nucl. Fusion 201757036027

[3] S. von Goeler et al., Phys. Rev. Lett. 197433 p.1201

[4] O. Sauter et al., Phys. Rev. Lett. 200288105001

[5] I. Chapman et al., Nucl. Fusion 201050102001

[6] C. Angioni et al., Nucl. Fusion 200343455

[7] A. Mück et al., Plasma Phys. Contr. Fus. 2005471633

[8] M. Lennholm et al., Phys. Rev. Lett. 2009102115004

[9] G. Witvoet et al., Nucl. Fusion 201252074005

[10] T. Goodman et al., Phys. Rev. Lett. 2011106245002

[11] M. Lauret et al., Nucl. Fusion 201252062002

[12] S. Nowak et al., Nucl. Fusion 201454033003

[13] R. White et al., Phys. Rev. Lett. 198962539

[14] F. Porcelli et al., Plasma Phys. Contr. Fus. 1996382163

[15] J. Graves et al., Phys. Rev. Lett. 2009102065005

[16] J. Graves et al., Plasma Phys. Contr. Fus. 201557014033

[17] J. Graves et al., Nat. Commun. 20123624

[18] I. Chapman et al., Nucl. Fusion 201252063006

[19] M. Lennholm et al., Nucl. Fusion, 201151073032

[20] L.-G. Eriksson et al 2004 Phys. Rev. Lett. 92235004

[21] L.-G. Eriksson et al 2006 Nucl. Fusion 46 S951

[22] F. Romanelli et al., Nucl. Fusion 201353104002

[23] R. Neu et al., 2013 Phys. Plasmas 20056111

[24] G. Matthews et al 2013 J. Nucl. Mater. 438 S2-10

[25] E. Lerche et al., Nucl. Fusion 201656036022

[26] M. Goniche et al., Plasma Phys. Contr. Fus. 201759 055001

[27] E. Joffrin et al 2014 Nuclear Fusion 54013011

[28] M. Beurskens et al., Plasma Phys. Contr. Fus. 201355 124043

[29] R. Neu et al., Plasma Phys. Contr. Fus. 200749 B59-B70

[30] T. Pütterich et al., Plasma Phys. Contr. Fus. 201355 124036

[31] R. Dux et al., Journal Nucl. Mat. 2009 390-391 858

[32] M. Mantsinen et al., these proceedings

[33] T.H. Stix, "Waves in Plasmas" (1992) AIP, New York

[34] A. Murari et al., Nucl. Fusion 201656076008

[35] M. Lennholm et al. accepted in Fus. Eng. \& Design (2017). 